Int. J. Open Problems Compt. Math., Vol. 6, No. 2, June 2013

ISSN 1998-6262; Copyright (CICSRS Publication, 2012

www.i-csrs.org

\title{
New Fractional Results For a Boundary \\ Value Problem With Caputo Derivative
}

\author{
${ }^{1}$ Mohamed Houas and ${ }^{2}$ Zoubir Dahmani \\ ${ }^{1}$ University of Khemis Miliana, Algeria \\ e-mail:mhouas@yahoo.fr \\ ${ }^{2}$ Laboratory of Pure and Applied Mathematics, LPAM, \\ Faculty SEI, UMAB University of Mostaganem \\ e-mail: zzdahmani@yahoo.fr
}

\begin{abstract}
In this paper, we consider three point boundary value problem for fractional differential equations of order $1<\alpha<2$. We establish new conditions for the existence and uniqueness of solutions by using Banach fixed point theorem. We also generate other existence results using Scheafer and Krasnoselskii fixed point theorems.
\end{abstract}

Keywords: Boundary value problem, Caputo derivative, Fixed point theorem. 2010 Mathematics Subject Classification: 34A08, 34A12, 34B25.

\section{Introduction}

The theory of differential equations of fractional order arises in many scientific disciplines, such as physics, chemistry, electrochemistry, control theory, image and signal processing, biophysics. For more details, we refer the reader to $[3,5,9,10,12,13,14,15,17]$ and references therein. There has been a significant progress in the investigation of these equations in recent years, (see $[4,6,7,12,16])$. More recently, some basic theory for the initial boundary value problems of fractional differential equations has been discussed in $[1,2,11,12]$. Motivated by the classical problem (1.1)-(1.2) in [8], this paper deals with the 
existence of solution for three point boundary value problems for the following problem

$$
\begin{aligned}
& D^{\alpha} x(t)+f(t, x(t))=0, t \in J, 1<\alpha<2, \\
& x(0)-\beta_{1} x^{\prime}(0)=0, x(1)-\beta_{2} x^{\prime}(\eta)=0,0<\eta<1,
\end{aligned}
$$

where $D^{\alpha}$ denote the fractional derivative of order $\alpha$ in the sense of Caputo, $J=[0,1], \beta_{1}, \beta_{2}$ are real constants with $\beta_{1}+1 \neq \beta_{2}, f$ is a continuous function on $J \times \mathbb{R}$.

\section{Preliminaries}

In the following, we give the necessary notation and basic definitions which will be used in this paper.

Definition 2.1: The Riemann-Liouville fractional integral operator of order $\alpha \geq 0$, for a continuous function $f$ on $[0, \infty[$ is defined as

$$
\begin{gathered}
J^{\alpha} f(t)=\frac{1}{\Gamma(\alpha)} \int_{0}^{t}(t-\tau)^{\alpha-1} f(\tau) d \tau ; \quad \alpha>0, t>0, \\
J^{0} f(t)=f(t),
\end{gathered}
$$

where $\Gamma(\alpha):=\int_{0}^{\infty} e^{-u} u^{\alpha-1} d u$.

Definition 2.2: The fractional derivative of $f \in C^{n}([0, \infty[)$ in the Caputo's sense is defined as

$$
D^{\alpha} f(t)= \begin{cases}\frac{1}{\Gamma(n-\alpha)} \int_{0}^{t}(t-\tau)^{n-\alpha-1} f^{(n)}(\tau) d \tau, & n-1<\alpha<n, n \in N^{*} \\ \frac{d^{n}}{d t^{n}} f(t), & \alpha=n .\end{cases}
$$

Details on Caputo's derivative can be found in $[12,15]$.

Let us now introduce $C(J, \mathbb{R})$ the Banach space of all continuous functions from $J$ into $\mathbb{R}$ with the norm $\|x\|=\sup _{t \in J}|x(t)|$.

We give the following lemmas [11]:

Lemma 2.1 For $\alpha>0$, the general solution of the fractional differential $D^{\alpha} x=0$ is given by

$$
x(t)=c_{0}+c_{1} t+c_{2} t^{2}+\ldots c_{n-1} t^{n-1},
$$

where $c_{i} \in \mathbb{R}, i=0,1,2, \ldots n-1, n=[\alpha]+1$.

Lemma 2.2 Let $\alpha>0$, then

$$
J^{\alpha} D^{\alpha} x(t)=x(t)+c_{0}+c_{1} t+c_{2} t^{2}+\ldots c_{n-1} t^{n-1},
$$

for some $c_{i} \in \mathbb{R}, i=0,1,2, \ldots n-1, n=[\alpha]+1$. 
We need also the following lemma:

Lemma 2.3 Let $1<\alpha<2$. A solution of (1) is given by:

$$
\begin{aligned}
x(t)= & \frac{-1}{\Gamma(\alpha)} \int_{0}^{t}(t-\tau)^{\alpha-1} f(\tau, x(\tau)) d \tau+\frac{\beta_{1}+t}{\left(\beta_{1}+1-\beta_{2}\right) \Gamma(\alpha)} \int_{0}^{1}(1-\tau)^{\alpha-1} f(\tau, x(\tau)) d \tau \\
& -\frac{\beta_{2}\left(\beta_{1}+t\right)}{\left(\beta_{1}+1-\beta_{2}\right) \Gamma(\alpha-1)} \int_{0}^{\eta}(\eta-\tau)^{\alpha-2} f(\tau, x(\tau)) d \tau .
\end{aligned}
$$

To present our main results, we need to define the following integral operator $F: C(J, \mathbb{R}) \rightarrow C(J, \mathbb{R})$ as follows:

$$
\begin{aligned}
\phi x(t)= & \frac{-1}{\Gamma(\alpha)} \int_{0}^{t}(t-\tau)^{\alpha-1} f(\tau, x(\tau)) d \tau+\frac{\beta_{1}+t}{\left(\beta_{1}+1-\beta_{2}\right) \Gamma(\alpha)} \int_{0}^{1}(1-\tau)^{\alpha-1} f(\tau, x(\tau)) d \tau \\
& -\frac{\beta_{2}\left(\beta_{1}+t\right)}{\left(\beta_{1}+1-\beta_{2}\right) \Gamma(\alpha-1)} \int_{0}^{\eta}(\eta-\tau)^{\alpha-2} f(\tau, x(\tau)) d \tau
\end{aligned}
$$

\section{Main Results}

For the forthcoming analysis, we need the following assumptions:

(A1): There exists a constant $k>0$ such that $|f(t, x)-f(t, y)| \leq k|x-y|$, for each $t \in J$ and all $x, y \in \mathbb{R}$.

(A2): The function $f: J \times \mathbb{R} \rightarrow \mathbb{R}$ is continuous.

(A3): There exists a constant $N>0$, such that $|f(t, x)| \leq N$, for each $t \in J$ and all $x \in \mathbb{R}$.

Our first result is given by:

Theorem 3.1 Assume that the hypothesis (A1) holds.

If

$$
k \frac{\left(\left|\beta_{1}+1-\beta_{2}\right|+\left|\beta_{1}+1\right|+\alpha \eta\left|\beta_{2} \beta_{1}+\beta_{2}\right|\right)}{\Gamma(\alpha+1)\left|\beta_{1}+-1 \beta_{2}\right|}<1
$$

then the problem (1) has a unique solution on J.

Proof: We shall prove that $\phi$ is contraction mapping on $C(J, \mathbb{R})$.

Let $x, y \in C(J, \mathbb{R})$. Then, for each $t \in J$, we can write 


$$
\begin{aligned}
\mid & \phi x(t)-\phi y(t)|=| \frac{-1}{\Gamma(\alpha)} \int_{0}^{t}(t-\tau)^{\alpha-1} f(\tau, x(\tau)) \\
& +\frac{\beta_{1}+t}{\left(\beta_{1}+1-\beta_{2}\right) \Gamma(\alpha)} \int_{0}^{1}(1-\tau)^{\alpha-1} f(\tau, x(\tau)) d \tau \\
& -\frac{\beta_{2}\left(\beta_{1}+t\right)}{\left(\beta_{1}+1-\beta_{2}\right) \Gamma(\alpha-1)} \int_{0}^{\eta}(\eta-\tau)^{\alpha-2} f(\tau, x(\tau)) d \tau \\
& +\frac{1}{\Gamma(\alpha)} \int_{0}^{t}(t-\tau)^{\alpha-1} f(\tau, x(\tau)) d \tau \\
& -\frac{\beta_{1}+t}{\left(\beta_{1}+1-\beta_{2}\right) \Gamma(\alpha)} \int_{0}^{1}(1-\tau)^{\alpha-1} f(\tau, x(\tau)) d \tau \\
& +\frac{\beta_{2}\left(\beta_{1}+t\right)}{\left(\beta_{1}+1-\beta_{2}\right) \Gamma(\alpha-1)} \int_{0}^{\eta}(\eta-\tau)^{\alpha-2} f(\tau, x(\tau)) d \tau \\
+ & \frac{\beta_{2}\left(\beta_{1}+t\right)}{\left(\beta_{1}+1-\beta_{2}\right) \Gamma(\alpha-1)} \int_{0}^{\eta}(\eta-\tau)^{\alpha-2} f(\tau, x(\tau)) d \tau \mid
\end{aligned}
$$

Thanks to $(A 1)$, we obtain

$$
\begin{aligned}
& |\phi(x)(t)-\phi(y)(t)| \leq \frac{1}{\Gamma(\alpha)} \int_{0}^{t}(t-\tau)^{\alpha-1}|f(\tau, x(\tau))-f(\tau, y(\tau))| d \tau \\
& \quad+\frac{\left|\beta_{1}+t\right|}{\left|\beta_{1}+1-\beta_{2}\right| \Gamma(\alpha)} \int_{0}^{1}(1-\tau)^{\alpha-1}|f(\tau, x(\tau))-f(\tau, y(\tau))| d \tau \\
& +\frac{\left|\beta_{2} \beta_{1}+\beta_{2} t\right|}{\left|\beta_{1}+1-\beta_{2}\right| \Gamma(\alpha-1)} \int_{0}^{\eta}(\eta-\tau)^{\alpha-2}|f(\tau, x(\tau))-f(\tau, y(\tau))| d \tau \\
& \leq \frac{k\|x-y\|}{\Gamma(\alpha)} \int_{0}^{t}(t-\tau)^{\alpha-1} d \tau+\frac{\left|\beta_{1}+1\right| k\|x-y\|}{\left|\beta_{1}+1-\beta_{2}\right| \Gamma(\alpha)} \int_{0}^{1}(1-\tau)^{\alpha-1} d \tau \\
& \quad+\frac{\left|\beta_{2} \beta_{1}+\beta_{2}\right| k\|x-y\|}{\left|\beta_{1}+1-\beta_{2}\right| \Gamma(\alpha-1)} \int_{0}^{\eta}(\eta-\tau)^{\alpha-2} d \tau \\
& \quad \leq k \frac{\left(\left|\beta_{1}+1-\beta_{2}\right|+\left|\beta_{1}+1\right|+\alpha \eta\left|\beta_{2} \beta_{1}+\beta_{2}\right|\right)}{\Gamma(\alpha+1)\left|\beta_{1}+1-\beta_{2}\right|}\|x-y\| .
\end{aligned}
$$

Therefore,

$\|\phi(x)-\phi(y)\| \leq k \frac{\left(\left|\beta_{1}+1-\beta_{2}\right|+\left|\beta_{1}+1\right|+\alpha \eta\left|\beta_{2} \beta_{1}+\beta_{2}\right|\right)}{\Gamma(\alpha+1)}\|x-y\|$. 
Thanks to (8), we conclude that $\phi$ is a contraction mapping. Hence by Banach fixed point theorem, there exists a unique fixed point $x \in C(J, \mathbb{R})$, which is a solution of (1).

The second result is the following.

Theorem 3.2 Suppose that the conditions (A2) and (A3) are satisfied. Then the problem (1) has at least a solution on $J$.

Proof: We use Scheafer's fixed point theorem to prove that $\phi$ has at least a fixed point on $C(J, \mathbb{R})$ :

Step1: The operator $\phi$ is continuous on $C(J, \mathbb{R})$ : Let $x_{n}$ be a sequence such that $x_{n} \rightarrow x$ in $C(J, \mathbb{R})$. Then, for each $t \in J$, we have:

$$
\begin{gathered}
\left|\phi\left(x_{n}\right)(t)-\phi(x)(t)\right|=\mid \frac{-1}{\Gamma(\alpha)} \int_{0}^{t}(t-\tau)^{\alpha-1} f\left(\tau, x_{n}(\tau)\right) d \tau \\
+\frac{\beta_{1}+t}{\left(\beta_{1}+1-\beta_{2}\right) \Gamma(\alpha)} \int_{0}^{1}(1-\tau)^{\alpha-1} f\left(\tau, x_{n}(\tau)\right) d \tau \\
-\frac{\beta_{2}\left(\beta_{1}+t\right)}{\left(\beta_{1}+1-\beta_{2}\right) \Gamma(\alpha-1)} \int_{0}^{\eta}(\eta-\tau)^{\alpha-2} f\left(\tau, x_{n}(\tau)\right) d \tau \\
\quad+\frac{1}{\Gamma(\alpha)} \int_{0}^{t}(t-\tau)^{\alpha-1} f(\tau, x(\tau)) d \tau \\
-\frac{\beta_{1}+t}{\left(\beta_{1}+1-\beta_{2}\right) \Gamma(\alpha)} \int_{0}^{1}(1-\tau)^{\alpha-1} f(\tau, x(\tau)) d \tau \\
+\frac{\beta_{2}\left(\beta_{1}+1\right)}{\left(\beta_{1}+1-\beta_{2}\right) \Gamma(\alpha-1)} \int_{0}^{\eta}(\eta-\tau)^{\alpha-2} f(\tau, x(\tau)) d \tau \mid
\end{gathered}
$$

Therefore,

$$
\begin{aligned}
& \left|\phi\left(x_{n}\right)(t)-\phi(x)(t)\right| \leq \frac{1}{\Gamma(\alpha)} \int_{0}^{t}(t-\tau)^{\alpha-1}\left|f\left(\tau, x_{n}\right)-f(\tau, x)\right| d \tau \\
& +\frac{\left|\beta_{1}+1\right|}{\left|\beta_{1}+1-\beta_{2}\right| \Gamma(\alpha)} \int_{0}^{1}(1-\tau)^{\alpha-1}\left|f\left(\tau, x_{n}\right)-f(\tau, x)\right| d \tau \\
& +\frac{\left|\beta_{2} \beta_{1}+\beta_{2}\right|}{\left|\beta_{1}+1-\beta_{2}\right| \Gamma(\alpha-1)} \int_{0}^{\eta}(\eta-\tau)^{\alpha-2}\left|f\left(\tau, x_{n}\right)-f(\tau, x)\right| d \tau .
\end{aligned}
$$

Thanks to $(A 2)$, we obtain 


$$
\left\|\phi\left(x_{n}\right)-\phi(x)\right\| \rightarrow 0, n \rightarrow \infty .
$$

Step2: The operator $\phi$ maps bounded sets into bounded sets in $C(J, \mathbb{R})$ : So, let us take $x \in B_{\mu}=\{x \in C(J, \mathbb{R}) ;\|x\| \leq \mu, \mu>0\}$.

By (A3), we have:

$$
\begin{gathered}
|\phi x(t)| \leq \frac{1}{\Gamma(\alpha)} \int_{0}^{t}(t-\tau)^{\alpha-1}|f(\tau, x(\tau))| d \tau \\
+\frac{\left|\beta_{1}+1\right|}{\left|\beta_{1}+1-\beta_{2}\right| \Gamma(\alpha)} \int_{0}^{1}(1-\tau)^{\alpha-1}|f(\tau, x(\tau))| d \tau \\
+\frac{\left|\beta_{2} \beta_{1}+\beta_{2}\right|}{\left|\beta_{1}+1-\beta_{2}\right| \Gamma(\alpha-1)} \int_{0}^{\eta}(\eta-\tau)^{\alpha-2}|f(\tau, x(\tau))| d \tau \\
\leq \frac{N}{\Gamma(\alpha)} \int_{0}^{t}(t-\tau)^{\alpha-1} d \tau+\frac{N\left|\beta_{1}+1\right|}{\left|\beta_{1}+1-\beta_{2}\right| \Gamma(\alpha)} \int_{0}^{1}(1-\tau)^{\alpha-1} d \tau \\
+\frac{N\left|\beta_{2} \beta_{1}+\beta_{2}\right|}{\left|\beta_{1}+1-\beta_{2}\right| \Gamma(\alpha-1)} \int_{0}^{\eta}(\eta-\tau) d \tau \\
\leq \frac{N\left|\beta_{1}+1\right|}{\Gamma(\alpha+1)}+\frac{\alpha \eta N\left|\beta_{2} \beta_{1}+\beta_{2}\right|}{\Gamma(\alpha+1)\left|\beta_{1}+1-\beta_{2}\right|}+\frac{\alpha(\alpha+1)}{\Gamma}
\end{gathered}
$$

Thus,

$$
\|\phi(x)\| \leq \frac{N\left|\beta_{1}+1-\beta_{2}\right|+N\left|\beta_{1}+1\right|+\alpha \eta N\left|\beta_{2} \beta_{1}+\beta_{2}\right|}{\Gamma(\alpha+1)\left|\beta_{1}+1-\beta_{2}\right|} .
$$

And consequently,

$$
\|\phi(x)\|<\infty .
$$

Step3: The operator $\phi$ maps bounded sets into equicontinuous sets of $C(J, \mathbb{R}):$

Let $t_{1}, t_{2} \in J ; t_{2}<t_{1}, x \in B_{\mu}$. Then, we have

$$
\begin{gathered}
\left|\phi x\left(t_{1}\right)-\phi x\left(t_{2}\right)\right|=\mid \frac{-1}{\Gamma(\alpha)} \int_{0}^{t_{1}}\left(t_{1}-\tau\right)^{\alpha-1} f(\tau, x(\tau)) d \tau \\
+\frac{\beta_{1}+t_{1}}{\left(\beta_{1}+1-\beta_{2}\right) \Gamma(\alpha)} \int_{0}^{1}(1-\tau)^{\alpha-1} f(\tau, x(\tau)) d \tau \\
-\frac{\beta_{2}\left(\beta_{1}+t_{1}\right)}{\left(\beta_{1}+1-\beta_{2}\right) \Gamma(\alpha-1)} \int_{0}^{\eta}(\eta-\tau)^{\alpha-2} f(\tau, x(\tau)) d \tau
\end{gathered}
$$




$$
\begin{gathered}
+\frac{1}{\Gamma(\alpha)} \int_{0}^{t_{2}}\left(t_{2}-\tau\right)^{\alpha-1} f(\tau, x(\tau)) d \tau \\
-\frac{\beta_{1}+t_{2}}{\left(\beta_{1}+1-\beta_{2}\right) \Gamma(\alpha)} \int_{0}^{1}(1-\tau)^{\alpha-1} f(\tau, x(\tau)) \\
+\frac{\beta_{2}\left(\beta_{1}+t_{2}\right)}{\left(\beta_{1}+1-\beta_{2}\right) \Gamma(\alpha-1)} \int_{0}^{\eta}(\eta-\tau)^{\alpha-2} f(\tau, x(\tau)) d \tau \mid .
\end{gathered}
$$

Therefore,

$$
\begin{aligned}
\mid \phi x\left(t_{1}\right)- & \phi x\left(t_{2}\right)\left|\leq \frac{1}{\Gamma(\alpha)} \int_{0}^{t_{2}}\left(t_{1}-\tau\right)^{\alpha-1}-\left(t_{2}-\tau\right)^{\alpha-1}\right| f(\tau, x(\tau)) \mid d \tau \\
& +\frac{t_{1}-t_{2}}{\left|\beta_{1}+1-\beta_{2}\right| \Gamma(\alpha)} \int_{0}^{1}(1-\tau)^{\alpha-1}|f(\tau, x(\tau))| d \tau \\
& +\frac{\left|\beta_{2}\right|\left(t_{1}-t_{2}\right)}{\left|\beta_{1}+1-\beta_{2}\right| \Gamma(\alpha-1)} \int_{0}^{\eta}(\eta-\tau)|f(\tau, x(\tau))| d \tau \\
& +\frac{1}{\Gamma(\alpha)} \int_{t_{2}}^{t_{1}}\left(t_{1}-\tau\right)^{\alpha-1}|f(\tau, x(\tau))| d \tau
\end{aligned}
$$

Thus,

$$
\begin{gathered}
\left|\phi x\left(t_{1}\right)-\phi x\left(t_{2}\right)\right| \leq \frac{\left(N+N \alpha \eta\left|\beta_{2}\right|\right)}{\Gamma(\alpha+1)\left|\beta_{1}+1-\beta_{2}\right|}\left(t_{1}-t_{2}\right) \\
+\frac{2 N}{\Gamma(\alpha+1)}\left(t_{1}-t_{2}\right)^{\alpha}+\frac{N}{\Gamma(\alpha+1)}\left(t_{2}^{\alpha}-t_{1}^{\alpha}\right) .
\end{gathered}
$$

As $t_{2} \rightarrow t_{1}$, the right-hand side of (20) tends to zero. Then, combining the Steps 1,2,3 with Arzela-Ascoli theorem, we conclude that $\phi$ is completely continuous.

Step4: The set

$$
\Omega=\{x \in C(J, \mathbb{R}), x=\lambda \phi(x), 0<\lambda<1\}
$$

is bounded:

Let $x \in \Omega$, then $x=\lambda \phi(x)$, for some $0<\lambda<1$. Hence, for $t \in J$, we have:

$$
x(t)=\lambda\left[\frac{-1}{\Gamma(\alpha)} \int_{0}^{t}(t-\tau)^{\alpha-1} f(\tau, x(\tau))+\frac{\beta_{1}+t}{\left(\beta_{1}+1-\beta_{2}\right) \Gamma(\alpha)} \int_{0}^{1}(1-\tau)^{\alpha-1} f(\tau, x(\tau)) d \tau\right.
$$




$$
\left.-\frac{\beta_{2}\left(\beta_{1}+t\right)}{\left(\beta_{1}+1-\beta_{2}\right) \Gamma(\alpha-1)} \int_{0}^{\eta}(\eta-\tau)^{\alpha-2} f(\tau, x(\tau)) d \tau\right] .
$$

Thanks to (A3), we can write

$$
\begin{gathered}
\frac{1}{\lambda}|x(t)| \leq \frac{1}{\Gamma(\alpha)} \int_{0}^{t}(t-\tau)^{\alpha-1}|f(\tau, x(\tau))| d \tau \\
+\frac{\left|\beta_{1}+1\right|}{\left|\beta_{1}+1-\beta_{2}\right| \Gamma(\alpha)} \int_{0}^{1}(1-\tau)^{\alpha-1}|f(\tau, x)| d \tau \\
+\frac{\left|\beta_{2} \beta_{1}+\beta_{2}\right|}{\left|\beta_{1}+1-\beta_{2}\right| \Gamma(\alpha-1)} \int_{0}^{\eta}(\eta-\tau)^{\alpha-2}|f(\tau, x(\tau))| d \tau .
\end{gathered}
$$

Therefore,

$$
\|x\| \leq \lambda \frac{N\left(\left|\beta_{1}+1-\beta_{2}\right|+\left|\beta_{1}+1\right|+\alpha \eta\left|\beta_{2} \beta_{1}+\beta_{2}\right|\right)}{\Gamma(\alpha+1)\left|\beta_{1}+1-\beta_{2}\right|} .
$$

Hence,

$$
\|\phi(x)\|<\infty .
$$

This shows that the set $\Omega$ is bounded.

As consequence of Schaefer's fixed point theorem, we deduce that $\phi$ has at least a fixed point, which is a solution of (1).

Now, we use Krasnselskii theorem [11] to prove the following result:

Theorem 3.3 Assume that the hypotheses (A1)-(A2)-(A3) hold, such that

$$
k<\Gamma(\alpha+1) .
$$

If there exists $\sigma \in \mathbb{R}$ such that

$$
\frac{N\left(\left|\beta_{1}+1\right|+\alpha \eta\left|\beta_{2} \beta_{1}+\beta_{2}\right|\right)}{\left|\beta_{1}+1-\beta_{2}\right| \Gamma(\alpha+1)} \leq \sigma,
$$

then the problem (1) has at least a solution on $J$.

Proof: Suppose that (27) holds and let us take

$$
\phi x(t):=H_{1} x(t)+H_{2} x(t),
$$

where

$$
H_{1} x(t):=\frac{-1}{\Gamma(\alpha)} \int_{0}^{t}(t-\tau)^{\alpha-1} f(\tau, x(\tau)) d \tau
$$


and

$$
\begin{aligned}
& H_{2} x(t):=\frac{\beta_{1}+t}{\left(\beta_{1}+1-\beta_{2}\right) \Gamma(\alpha)} \int_{0}^{1}(1-\tau)^{\alpha-1} f(\tau, x(\tau)) d \tau \\
& -\frac{\beta_{2}\left(\beta_{1}+t\right)}{\left(\beta_{1}+1-\beta_{2}\right) \Gamma(\alpha-1)} \int_{0}^{\eta}(\eta-\tau)^{\alpha-2} f(\tau, x(\tau)) d \tau .
\end{aligned}
$$

$\left(1^{*}\right)$ : We shall prove that $H_{1}$ is a contraction mapping: Let $x, y \in C(J, \mathbb{R})$. Then, for each $t \in J$, we can write

$$
\begin{aligned}
& \left|H_{1} x(t)-H_{1} y(t)\right|=\mid-\frac{1}{\Gamma(\alpha)} \int_{0}^{t}(t-\tau)^{\alpha-1} f(\tau, x(\tau)) d \tau \\
& \quad+\frac{1}{\Gamma(\alpha)} \int_{0}^{t}(t-\tau)^{\alpha-1} f(\tau, y(\tau)) d \tau \\
& \leq \frac{1}{\Gamma(\alpha)} \int_{0}^{t}(t-\tau)^{\alpha-1}|f(\tau, x(\tau))-f(\tau, y(\tau))| d \tau .
\end{aligned}
$$

By (A1), we get

$$
\left\|H_{1}(x)-H_{1}(y)\right\| \leq \frac{k}{\Gamma(\alpha+1)}\|x-y\| .
$$

Using the condition (26) we conclude that $H_{1}$ is a contraction mapping. $\left(2^{*}\right)$ : We shall prove that $H_{2}$ is continuous:

Let $x_{n}$ be sequence such that $x_{n} \rightarrow x$ in $C(J, \mathbb{R})$. Then for each $t \in J$,

$$
\begin{aligned}
& \left|H_{2} x_{n}(t)-H_{2} x(t)\right|=\left|\frac{\beta_{1}+t}{\left(\beta_{1}+1-\beta_{2}\right) \Gamma(\alpha)} \int_{0}^{1}(1-\tau)^{\alpha-1} f\left(\tau, x_{n}(\tau)\right)\right| \\
& -\frac{\beta_{2}\left(\beta_{1}+t\right)}{\left(\beta_{1}+1-\beta_{2}\right) \Gamma(\alpha-1)} \int_{0}^{\eta}(\eta-\tau)^{\alpha-1} f\left(\tau, x_{n}(\tau)\right) d \tau \\
& \quad-\frac{\beta_{1}+t}{\left(\beta_{1}+1-\beta_{2}\right) \Gamma(\alpha)} \int_{0}^{1}(1-\tau)^{\alpha-1} f(\tau, x(\tau)) d \tau \\
& \quad+\frac{\beta_{2}\left(\beta_{1}+t\right)}{\left(\beta_{1}+1-\beta_{2}\right) \Gamma(\alpha-1)} \int_{0}^{\eta}(\eta-\tau)^{\alpha-1} f(\tau, x(\tau)) d \tau \mid \\
& \leq \frac{\left|\beta_{1}+1\right|}{\left|\beta_{1}+1-\beta_{2}\right| \Gamma(\alpha)} \int_{0}^{1}(1-\tau)^{\alpha-1}\left|f\left(\tau, x_{n}(\tau)\right)-f(\tau, x(\tau))\right| d \tau \\
& \frac{\left|\beta_{2} \beta_{1}+\beta_{2}\right|}{\left|\beta_{1}+1-\beta_{2}\right| \Gamma(\alpha-1)} \int_{0}^{\eta}(\eta-\tau)^{\alpha-2}\left|f\left(\tau, x_{n}(\tau)\right)-f(\tau, x(\tau))\right| d \tau
\end{aligned}
$$




$$
\leq \frac{\left|\beta_{1}+1\right|+\alpha \eta\left|\beta_{2} \beta_{1}+\beta_{2}\right|}{\left|\beta_{1}+1-\beta_{2}\right| \Gamma(\alpha+1)}\left\|f\left(., x_{n}\right)-f(., x)\right\|
$$

Since $f$ is a continuous function, we have

$$
\left\|H_{2}\left(x_{n}\right)-H_{2}(x)\right\| \rightarrow 0, n \rightarrow \infty .
$$

$\left(3^{*}\right)$ : Now, we prove that $H_{2}$ maps bounded sets into bounded sets of $C(J, \mathbb{R}):$ Let $x \in B_{\sigma}$. It is clear that

$$
\begin{gathered}
\left|H_{2} x(t)\right|=\mid \frac{\beta_{1}+t}{\left(\beta_{1}+1-\beta_{2}\right) \Gamma(\alpha)} \int_{0}^{1}(1-\tau)^{\alpha-1} f(\tau, x(\tau)) d \tau \\
-\frac{\beta_{2}\left(\beta_{1}+t\right)}{\left(\beta_{1}+1-\beta_{2}\right) \Gamma(\alpha-1)} \int_{0}^{\eta}(\eta-\tau)^{\alpha-1} f(\tau, x(\tau)) d \tau \mid .
\end{gathered}
$$

Therefore,

$$
\begin{aligned}
& \left|H_{2} x(t)\right| \leq \frac{\left|\beta_{1}+1\right|}{\left|\beta_{1}+1-\beta_{2}\right| \Gamma(\alpha)} \int_{0}^{1}(1-\tau)|f(\tau, x(\tau))| d \tau \\
& +\frac{\left|\beta_{2} \beta_{1}+\beta_{2}\right|}{\left|\beta_{1}+1-\beta_{2}\right| \Gamma(\alpha-1)} \int_{0}^{\eta}(\eta-\tau)^{\alpha-2}|f(\tau, x(\tau))| d \tau
\end{aligned}
$$

Tanks to (A3), we can write:

$$
\begin{gathered}
\left\|\quad H_{2}(x)\right\| \leq \frac{N\left|\beta_{1}+1\right|}{\left|\beta_{1}+1-\beta_{2}\right| \Gamma(\alpha)} \int_{0}^{1}(1-\tau)^{\alpha-1} d \tau \\
+\frac{N\left|\beta_{2} \beta_{1}+\beta_{2}\right|}{\left|\beta_{1}+1-\beta_{2}\right| \Gamma(\alpha-1)} \int_{0}^{\eta}(\eta-\tau)^{\alpha-2} d \tau
\end{gathered}
$$

Thus,

$$
\left\|H_{2}(x)\right\| \leq \frac{N\left|\beta_{1}+1\right|+\alpha \eta N\left|\beta_{2} \beta_{1}+\beta_{2}\right|}{\left|\beta_{1}+1-\beta_{2}\right| \Gamma(\alpha+1)} .
$$

Consequently,

$$
\left\|H_{2}(x)\right\|<\infty \text {. }
$$

$\left(4^{*}\right)$ : The operator $H_{2}$ maps bounded sets into equicontinuous sets of $C(J, \mathbb{R}):$

Let $t_{1}, t_{2} \in J ; t_{1}<t_{2}, x \in B_{\sigma}$. Then, we have

$$
\left|H_{2} x\left(t_{2}\right)-H_{2} x\left(t_{1}\right)\right|=\mid \frac{\beta_{1}+t_{2}}{\left(\beta_{1}+1-\beta_{2}\right) \Gamma(\alpha)} \int_{0}^{1}(1-\tau)^{\alpha-1} f(\tau, x(\tau)) d \tau
$$




$$
\begin{gathered}
-\frac{\beta_{2}\left(\beta_{1}+t_{2}\right)}{\left(\beta_{1}+1-\beta_{2}\right) \Gamma(\alpha-1)} \int_{0}^{\eta}(\eta-\tau)^{\alpha-2} f(\tau, x(\tau)) d \tau \\
-\frac{\beta_{1}+t_{1}}{\left(\beta_{1}+1-\beta_{2}\right) \Gamma(\alpha)} \int_{0}^{1}(1-\tau)^{\alpha-1} f(\tau, x(\tau)) d \tau \\
+\frac{\beta_{2}\left(\beta_{1}+t_{1}\right)}{\left(\beta_{1}+1-\beta_{2}\right) \Gamma(\alpha-1)} \int_{0}^{\eta}(\eta-\tau)^{\alpha-2} f(\tau, x(\tau)) d \tau \mid
\end{gathered}
$$

This implies that,

$$
\begin{gathered}
\left|H_{2} x\left(t_{2}\right)-H_{2} x\left(t_{1}\right)\right| \leq \frac{t_{2}-t_{1}}{\left|\beta_{1}+1-\beta_{2}\right| \Gamma(\alpha)} \int_{0}^{1}(1-\tau)^{\alpha-1}|f(\tau, x(\tau))| d \tau \\
\quad+\frac{\left|\beta_{2}\right| t_{2}-t_{1}}{\left|\beta_{1}+1-\beta_{2}\right| \Gamma(\alpha-1)} \int_{0}^{\eta}(\eta-\tau)^{\alpha-2}|f(\tau, x(\tau))| d \tau
\end{gathered}
$$

Hence, we have

$$
\left|H_{2} x\left(t_{2}\right)-H_{2} x\left(t_{1}\right)\right| \leq \frac{N}{\left|\beta_{1}+1-\beta_{2}\right| \Gamma(\alpha+1)}\left(t_{2}-t_{1}\right)+\frac{\alpha \eta N\left|\beta_{2}\right|}{\left|\beta_{1}+1-\beta_{2}\right| \Gamma(\alpha+1)}\left(t_{2}-t_{1}\right) .
$$

As $t_{1} \rightarrow t_{2}$ the right-hand side of (42) tends to zero. Then, as a consequence of steps $(2 *, 3 *, 4 *)$, we can conclude that $H_{2}$ is continuous and compact.

$\left(5^{*}\right)$ : Now, we shall prove that for any $x, y \in B_{\sigma}$, then $H_{1}(x)+H_{2}(y) \in B_{\sigma}$. So, let us take $x, y \in B_{\sigma}$. We have:

$$
\begin{aligned}
& \left|H_{1} x(t)+H_{2} y(t)\right|=\mid-\frac{1}{\Gamma(\alpha)} \int_{0}^{t}(t-\tau)^{\alpha-1} f(\tau, x(\tau)) d \tau \\
& +\frac{\beta+t}{\left(\beta_{1}+1-\beta_{2}\right) \Gamma(\alpha)} \int_{0}^{1}(1-\tau)^{\alpha-1} f(\tau, x(\tau)) d \tau \\
& -\frac{\beta_{2}\left(\beta_{1}+t\right)}{\left(\beta_{1}+1-\beta_{2}\right) \Gamma(\alpha-1)} \int_{0}^{\eta}(\eta-\tau)^{\alpha-2} f(\tau, x(\tau)) d \tau \mid \\
& \leq \frac{1}{\Gamma(\alpha)} \int_{0}^{t}(t-\tau)^{\alpha-1}|f(\tau, x(\tau))| d \tau \\
& +\frac{\left|\beta_{1}+1\right|}{\left|\beta_{1}+1-\beta_{2}\right| \Gamma(\alpha)} \int_{0}^{1}(1-\tau)^{\alpha-1}|f(\tau, x(\tau))| d \tau \\
& +\frac{\left|\beta_{2} \beta_{1}+\beta_{2}\right|}{\left|\beta_{1}+1-\beta_{2}\right| \Gamma(\alpha-1)} \int_{0}^{\eta}(\eta-\tau)^{\alpha-2}|f(\tau, x(\tau))| d \tau .
\end{aligned}
$$


By (A3), we have

$$
\begin{array}{cl}
\left\|\quad H_{1}(x)+H_{2}(y)\right\| \leq \frac{N\left|\beta_{1}+1\right|}{\left|\beta_{1}+1-\beta_{2}\right| \Gamma(\alpha)} \int_{0}^{1}(1-\tau)^{\alpha-1} d \tau \\
+\frac{N\left|\beta_{2} \beta_{1}+\beta_{2}\right|}{\left|\beta_{1}+1-\beta_{2}\right| \Gamma(\alpha-1)} \int_{0}^{\eta}(\eta-\tau)^{\alpha-2} d \tau .
\end{array}
$$

Consequently,

$$
\left\|H_{1}(x)+H_{2}(y)\right\| \leq \frac{N\left(\left|\beta_{1}+1\right|+\alpha \eta\left|\beta_{2} \beta_{1}+\beta_{2}\right|\right)}{\left|\beta_{1}+1-\beta_{2}\right| \Gamma(\alpha+1)} .
$$

Using the condition (27), we conclude that $H_{1}(x)+H_{2}(y) \in B_{\sigma}$. As a consequence of Krasnoselskii's fixed point theorem we deduce that $\phi$ has a fixed point which is a solution of (1).

\section{Open Problems}

At the end, we pose the following open problems:

Open Problem 1: Using Riemann-Liouville fractional differential operator of order $\alpha$, under what conditions do Theorems 6,7 and Theorem 8 hold for $1<\alpha<2$ ?

Open Problem 2: Is it possible to generalize the above results for (1), where the derivative $D^{\alpha}$ is taken in the sense of Riemann-Liouville, $n<\alpha<n+1, n \in$ $\mathbb{N}$, and using Riemann-Liouville fractional initial conditions?

\section{References}

[1] A. Bashir. N. Juan, J. A. Aalsaedi: Existence and uniqueness of solutions for nonlinear fractional differential equations with non-separated type integral boundary conditions. Acta Mathematica Scientia. 31B(6) (2011), pp. 2122-2130.

[2] A. Belarbi, M. Benchohra, A. Ouahab: Uniqueness results of fractional functional differential equations with infinite delay in Frechet spaces. Appl. Anal. 85, (2006), No. 12, pp. 1459-1470.

[3] M.E. Bengrine, Z. Dahmani: Boundary value problems for fractional differential equations. Int. J. Open problems compt. Math. 5(4), (2012).

[4] D. Delbosco, L. Rodino: Existence and uniqueness for a nonlinear fractional differential equation. J. Math. Anal. Appl. 204 (3-4), (1996), pp. 429-440. 
[5] K. Diethelm, N.J. Ford: Analysis of fractiona differential equations. J. Math. Anal. Appl. 265(2), (2002), pp. 229-248.

[6] K. Diethelm, G. Walz: Numerical solution of fraction order differential equations by extrapolation. Numer. Algorithms. 16(3), (1998), pp. 231-253.

[7] A.M.A. El-Sayed: Nonlinear functional differential equations of arbitrary orders. Nonlinear Anal. 33(2), (1998), pp. 181-186.

[8] A, Guezane-Lakoud, S. Kelaiaia: Solvability of a nonlinear boundary value problem. TJNSA Journal. 4(4), (2011), pp. 247-261.

[9] R. Hilfer: Applications of fractional calculus in physics. World Scientific, Publishing Co., Inc., River Edge, NJ. (2000).

[10] A.A. Kilbas, S.A. Marzan: Nonlinear differential equation with the Caputo fraction derivative in the space of continuously differentiable functions. Differ. Equ. 41(1), (2005), pp. 84-89.

[11] V. Lakshmikantham, A.S. Vatsala: Basic theory of fractional differential equations. Nonlinear Anal. 69(8), (2008), pp. 2677-2682.

[12] F. Mainardi: Fractional calculus: some basic problem in continuum and statistical mechanics. Springer, Vienna. (1997).

[13] S.M. Momani, S.B. Hadid: Some comparison resultats for integro- fractional differential inequalities. J. Fract. Calc. 24, (2003), pp. 37-44.

[14] S.K. Ntouyas: Existence results for first order boundary value problems for fractional differential equations and inclusions with fractional integral boundary conditions. Journal of Fractional Calculus and Applications. 3(9), (2012), pp. 1-14.

[15] I. Podlubny, I. Petras, B.M. Vinager, P. O'leary, L. Dorcak: Analogue realizations of fractional-order controllers. Fractional order calculus and its applications. Nonlinear Dynam. 29(4), (2002), pp. 281-296.

[16] S. Zhang: Positive solutions for boundary-value problems of nonlinear fractional differential equations. Electron. J. Differential Equations. 2(36), (006), pp. 12-19.

[17] Z. Cui. P. Yu, Z. Mao: Existence of solutions for nonlocal boundary valueproblems of nonlinear fractional differential equations. Advances in Dynamical Systems and Applications. 7(1), (2012), pp. 31-40. 